University of Wollongong

Research Online

Australian Institute for Innovative Materials -

Papers

Australian Institute for Innovative Materials

$1-1-2018$

\title{
Magnesium Hydride Nanoparticles Self-Assembled on Graphene as Anode Material for High-Performance Lithium-Ion Batteries
}

\author{
Baoping Zhang \\ Fudan University \\ Guanglin Xia \\ Fudan University, University of Wollongong, guanglin@uow.edu.au \\ Dalin Sun \\ Fudan University, Shanghai Innovation Institute for Materials \\ Fang Fang \\ Fudan University \\ Xuebin Yu \\ Fudan University, Shanghai Innovation Institute for Materials, xyu@uow.edu.au
}

Follow this and additional works at: https://ro.uow.edu.au/aiimpapers

Part of the Engineering Commons, and the Physical Sciences and Mathematics Commons

Research Online is the open access institutional repository for the University of Wollongong. For further information contact the UOW Library: research-pubs@uow.edu.au 


\title{
Magnesium Hydride Nanoparticles Self-Assembled on Graphene as Anode Material for High-Performance Lithium-Ion Batteries
}

\begin{abstract}
$\mathrm{MgH} 2$ nanoparticles (NPs) uniformly anchored on graphene (GR) are fabricated based on a bottom-up self-assembly strategy as anode materials for lithium-ion batteries (LIBs). Monodisperse MgH $2 \mathrm{NPs}$ with an average particle size of $\sim 13.8 \mathrm{~nm}$ are self-assembled on the flexible GR, forming interleaved $\mathrm{MgH} 2$ /GR (GMH) composite architectures. Such nanoarchitecture could effectively constrain the aggregation of active materials, buffer the strain of volume changes, and facilitate the electron/lithium ion transfer of the whole electrode, leading to a significant enhancement of the lithium storage capacity of the GMH composite. Furthermore, the performances of GMH composite as anode materials for LIBs are enabled largely through robust interfacial interactions with poly(methyl methacrylate) (PMMA) binder, which plays multifunctional roles in forming a favorable solid-electrolyte interphase (SEI) film, alleviating the volume expansion and detachment of active materials, and maintaining the structural integrity of the whole electrode. As a result, these synergistic effects endow the obtained GMH composite with a significantly enhanced reversible capacity and cyclability as well as a good rate capability. The GMH composite with $50 \mathrm{wt} \% \mathrm{MgH} 2$ delivers a high reversible capacity of $946 \mathrm{~mA} \mathrm{~h} \mathrm{~g}-1$ at $100 \mathrm{~mA} \mathrm{~g}-1$ after 100 cycles and a capacity of $395 \mathrm{mAh} \mathrm{g}-1$ at a high current density of $2000 \mathrm{~mA} \mathrm{~g} \mathrm{-1}$ after 1000 cycles.

\section{Disciplines}

Engineering | Physical Sciences and Mathematics

\section{Publication Details}

Zhang, B., Xia, G., Sun, D., Fang, F. \& Yu, X. (2018). Magnesium Hydride Nanoparticles Self-Assembled on Graphene as Anode Material for High-Performance Lithium-Ion Batteries. ACS Nano, 12 (4), 3816-3824.
\end{abstract}




\section{Magnesium Hydride Nanoparticles Self-Assembled}

\section{on Graphene as Anode Material for High-}

\section{Performance Lithium-Ion Batteries}

Baoping Zhang, ${ }^{\dagger+}$ Guanglin Xia, ${ }^{*}+\$$, Dalin Sun, ${ }^{\dagger \neq}$ Fang Fang ${ }^{\dagger}$ and Xuebin Y $u^{* t \neq}$

${ }^{\dagger}$ Department of Materials Science, Fudan University, Shanghai 200433, China

${ }^{\S}$ Institute for Superconducting and Electronic Materials, University of Wollongong, North

Wollongong, NSW 2522, Australia

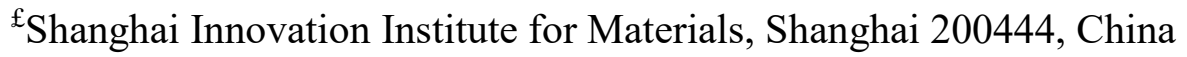

\section{Corresponding Author}

*E-mail: yuxuebin@fudan.edu.cn; guanglin@uow.edu.au 
ABSTRACT: $\mathrm{MgH}_{2}$ nanoparticles (NPs) uniformly anchored on graphene (GR) are fabricated based on a bottom-up self-assembly strategy as anode materials for lithium-ion batteries (LIBs). Monodisperse $\mathrm{MgH}_{2} \mathrm{NPs}$ with an average particle size of $\sim 13.8 \mathrm{~nm}$ are self-assembled on the flexible GR, forming interleaved $\mathrm{MgH}_{2} / \mathrm{GR}$ (GMH) composite architectures. Such nanoarchitecture could effectively constrain the aggregation of active materials, buffer the strain of volume changes, and facilitate the electron/lithium ion transfer of the whole electrode, leading to a significant enhancement of the lithium storage capacity of the GMH composite. Furthermore, the performances of GMH composite as anode materials for LIBs are enabled largely through robust interfacial interactions with poly (methyl methacrylate) (PMMA) binder, which plays multifunctional roles in forming a favorable solid-electrolyte interphase (SEI) film, alleviating the volume expansion and detachment of active materials, and maintaining the structural integrity of the whole electrode. As a result, these synergistic effects endow the obtained GMH composite with a significantly enhanced reversible capacity and cyclability, as well as a good rate capability. The GMH composite with $50 \mathrm{wt} \% \mathrm{MgH}_{2}$ delivers a high reversible capacity of $946 \mathrm{~mA} \mathrm{~h} \mathrm{~g}^{-1}$ at $100 \mathrm{~mA} \mathrm{~g}^{-1}$ after 100 cycles and a capacity of $395 \mathrm{mAh}$ $\mathrm{g}^{-1}$ at a high current density of $2000 \mathrm{~mA} \mathrm{~g}^{-1}$ after 1000 cycles.

KEYWORDS: lithium ion batteries; magnesium hydride; graphene; self-assembly; nanoarchitecture 
The rapidly expanding applications of lithium-ion batteries (LIBs) as power sources for portable electronic devices, electric vehicles and renewable energy storage systems have led to intensive researches on electrode materials with high electrochemical performances. ${ }^{1-3}$ To this end, kinds of promising candidates based on metals or semiconductors $\left(\right.$ e.g. $\left.\mathrm{Al}^{4}, \mathrm{Si}^{5}\right)$, transition metal oxides, ${ }^{6}$ sulphides, ${ }^{7,8}$ carbides and nitrides, ${ }^{9,} 10$ phosphides, ${ }^{11,12}$ and so forth, have been developed as anode materials. Specifically, Oumellal et al. ${ }^{13}$ pioneeringly proposed the use of metal hydrides as a concept of negative electrodes for LIBs, thus extending the field of promising conversion-type electrode materials. Among various metal hydrides, magnesium hydride $\left(\mathrm{MgH}_{2}\right)$ has drawn considerable attention due to its high theoretical gravimetric capacity (2038 $\left.\mathrm{mAh} \mathrm{g}^{-1}\right)$ and volumetric capacity $\left(2878 \mathrm{mAh} \mathrm{L}^{-1}\right)$, favorable voltage profile and low voltage hysteresis for conversion process, and more importantly, the abundant supply of raw materials in nature. ${ }^{14-17}$ However, the practical application of $\mathrm{MgH}_{2}$ in LIBs are plagued largely by its inevitable volume expansion/shrinkage $\left(\right.$ e.g. $\mathrm{MgH}_{2}(61.59 \AA) \rightarrow \mathrm{Mg}(46.46 \AA)+2 \mathrm{LiH}(2$ $\times 33.3 \AA$ ) ) during repeated lithium insertion/extraction processes, which finally gives rise to severe loss of capacity and poor cycling performance. For instance, the discharge capacity of bulk $\mathrm{MgH}_{2}$ electrode fades rapidly from initial $1480 \mathrm{mAh} \mathrm{g}^{-1}$ to less than $200 \mathrm{mAh} \mathrm{g}^{-1}$ after only 10 cycles. ${ }^{13}$ Fundamentally, the volume changes could cause the instability, rupture, reformation or incrassation of SEI film coating on active materials, consuming the 'live' lithium and electrolyte, and eventually battery dies from lithium exhaustion. Besides, those active materials (Mg-Li) could agglomerate or pulverize severely upon cycling, thus detaching from current collector. ${ }^{18,19}$

To address these issues, tremendous efforts have been made to improve the cycle performances of $\mathrm{MgH}_{2}$ electrode. One efficient strategy is to reduce the particle size of $\mathrm{MgH}_{2}$, which could 
alleviate the pulverization of bulk $\mathrm{MgH}_{2}$ particles upon cycling and enhance kinetics for storing lithium ions. For instance, Oumellal et al. ${ }^{15}$ reported an anode material based on $\mathrm{MgH}_{2}$ NPs/porous carbon composite synthesized by a ball milling treatment, and it delivered an improved cycle performance in comparison with bulk $\mathrm{MgH}_{2}$. However, due to the inevitable growth and aggregation of Mg-based NPs upon cycling, this improvement is limited to $500 \mathrm{mAh}$ $\mathrm{g}^{-1}$ after 25 cycles. Another efficient and practical approach to enhance the cycling stability of electrode is introducing polymeric binder, which can enhance electronic contact, favor to formation of stable SEI film, and buffer the strain of volume variation during cycling. ${ }^{20-23}$ However, it is noteworthy that the high reactivity of $\mathrm{MgH}_{2}$ particles makes the majority of traditional binders (e.g. Polyvinylidene fluoride, PVDF) unavailable or even harmful. Therefore, the basic principle for developing a binder in $\mathrm{MgH}_{2}$ electrode requires the binder inert to the electrode material. A successful case is adopting carboxymethylcellulose-formate (CMC-f) as a binder for $\mathrm{MgH}_{2}$-carbon electrodes, ${ }^{24}$ and it demonstrated an enhanced capacity retention with $542 \mathrm{mAh} \mathrm{g}^{-1}$ after 40 cycles. In spite of these favorable improvements, results are still fell far short of the theoretical promises. Therefore, further efforts are essential to achieve a homogeneous and stable dispersion of $\mathrm{MgH}_{2} \mathrm{NPs}$ as well as to explore much more suitable binders so as to develop a robust $\mathrm{MgH}_{2}$ electrode with high reversible capacity, stable cyclability and favorable rate capability for lithium storage.

Herein, combining these design criterions, we present a bottom-up self-assembly of $\mathrm{MgH}_{2} \mathrm{NPs}$ on GR as anode materials for LIBs. In this composite, monodisperse $\mathrm{MgH}_{2} \mathrm{NPs}$ with an average particle size of $\sim 13.8 \mathrm{~nm}$ are uniformly anchored on the ultrathin and flexible GR, thereby enhanced lithium storage performance can be brought out by the nanoparticles-graphene interactions derived at nanoscale. Such architecture of composite contributes to enhancing the 
interfacial contact, facilitating the electron/lithium ion transfer, constraining the self-aggregation of active materials, and buffering the strain of volume changes across the whole electrode during cycling. Furthermore, PMMA is proposed as a prospective binder for $\mathrm{MgH}_{2}$-based electrodes. The robust interfacial interactions with $\mathrm{MgH}_{2} / \mathrm{Li}_{\mathrm{x}} \mathrm{Mg}$ nanocrystals and GR of GMH composite, plays critical roles in alleviating the volume expansion and detachment of active materials, forming a favorable SEI film, and maintaining the structural integrity of electrode. As a result, GMH composite with PMMA binder is endowed with robust structure and excellent electrical conductivity as anode material for LIBs, which delivers a high specific capacity, favorable rate performance and largely enhanced cycling stability even at high rate.

\section{RESULTS AND DISCUSSION}

The GMH composite was fabricated by hydrogenation of di-butyl magnesium $\left(\left(\mathrm{C}_{4} \mathrm{H}_{9}\right)_{2} \mathrm{Mg}\right)$ in cyclohexane $\left(\mathrm{C}_{6} \mathrm{H}_{12}\right)$ under the structure-directing role of GR, as illustrated in Figure 1a. X-ray diffraction (XRD) confirms the formation of $\mathrm{MgH}_{2}$ (Figure 1b), and all diffraction peaks could be readily indexed to a pure tetragonal phase [space group: $\mathrm{P}_{2 / m n m}$ (136)] of $\mathrm{MgH}_{2}$ (JCPDS no. 12-0697).The morphology and nanostructure of the as-prepared composite was characterized by field-emission scanning electron microscopy (SEM) and transmission electron microscopy (TEM). Evidently, a large number of monodisperse $\mathrm{MgH}_{2}$ NPs with an average diameter of $\sim 13.8 \mathrm{~nm}$ (Figure S1) and free of aggregation are uniformly distributed on GR with a multilayer structure (Figure 1c-f). There is no isolated $\mathrm{MgH}_{2} \mathrm{NPs}$ in composite can be observed even after ultrasonic for $30 \mathrm{~min}$ to disperse the sample for TEM test, indicating the strong interactions between $\mathrm{MgH}_{2}$ and GR. The cross-sectional SEM image (Figure 1d) further displays that highdensity $\mathrm{MgH}_{2} \mathrm{NPs}$ are homogenously distributed throughout the surface and interleaved layers of GR. The selected-area electron diffraction (SAED) pattern (inset of Figure 1f) clearly 
demonstrates the polycrystalline structure of $\mathrm{MgH}_{2}$, which is consistent with the PXRD results and demonstrates the well-crystallized nature of $\mathrm{MgH}_{2}$. The high-resolution TEM (HRTEM) images and fast Fouriert ransform (FFT) pattern further verify that the obtained nanocrystals have a distinct lattice fringes with $d$-spacing of $2.23 \AA$, corresponding to the (200) planes of $\mathrm{MgH}_{2}$ (Figure 1g). The STEM image in Figure 1h and the corresponding elemental mapping of $\mathrm{C}$ and $\mathrm{Mg}$ (Figure 1i) further verified the uniform distribution of $\mathrm{MgH}_{2}$ on GR.

The porous structure and specific surface area of GMH composite were investigated by nitrogen isothermal adsorption (Figure S2a). GMH composite has a Brunauer-Emmett-Teller (BET) specific surface area of $151.5 \mathrm{~m}^{2} \mathrm{~g}^{-1}$ with a pore volume of $0.60 \mathrm{~cm}^{3} \mathrm{~g}^{-1}$ and an average pore diameter of $3.83 \mathrm{~nm}$ calculated by Barrett-Joyner-Halenda (BJH). Such highly hierarchical porous structure networks in association with large surface area can provide sufficient spaces for potential volume expansion, allow for easy diffusions of electrolyte to active sites, and facilitate fast transport of lithium ions, thus greatly improving lithium storage capacity and rate capability. ${ }^{25}$ The loading ratio of $\mathrm{MgH}_{2}$ in the typical composite was evaluated to be about $50 \mathrm{wt} \%$ by thermogravimetric analysis (TGA), as shown in Figure S2b.

The lithium-storage behaviors of GMH electrodes were illustrated by the galvanostatic charge/discharge tests and the specific capacities were tested based on the weight percentage of $\mathrm{MgH}_{2}$. Figure 2a presents the cycling performance of GMH composite with binder-free, PMMA and PVDF binder (denoted as GMH-BF, GMH-PMMA and GMH-PVDF, respectively) at a current density of $100 \mathrm{~mA} \mathrm{~g}^{-1}$ and a voltage ranging from 0.001 to $3 \mathrm{~V}$. As a comparison, the performance of commercial $\mathrm{MgH}_{2}$ electrode (CMH-PMMA, Figure S3) and the composite of GR and PMMA (GR-PMMA, Figure S4) were also explored under the same conditions. Firstly, it can be seen that the CMH-PMMA displays an initial discharge capacity of $1000 \mathrm{mAh} \mathrm{g}^{-1}$ and a 
severe capacity decay with only a capacity of $172 \mathrm{mAh} \mathrm{g}^{-1}$ left after 20 cycles, which is consistent with the results reported previously. ${ }^{24}$ On the sharp contrary, GMH-BF shows a high initial capacity of $4412 \mathrm{mAh} \mathrm{g}^{-1}$ and a reversible capacity of $1613 \mathrm{mAh} \mathrm{g}^{-1}$ at 20 cycles. It is noted that the high initial capacity is mainly ascribed to the formation of SEI layer on electrode surface or the irreversible side reactions, and the decomposition of electrolyte. ${ }^{13}$ Especially, based on the cycling performances of graphene (Figure S4), it is believed that although graphene in the composite contributes a high irreversible capacity (nearly $2000 \mathrm{mAh} \mathrm{g}^{-1}$ ) in the first discharge, its influence on capacity, especially at the high current rates, could be neglected after initial several cycles.

Since GMH electrode suffers from an apparent capacity decline after 20 cycles, two binders were introduced to reduce the loss of active materials and improve the cycling stability. Performance of GMH-PVDF in Figure 2a demonstrates that the most widely used PVDF binder largely lowers the reversible capacity of GMH owning to the reactivity between PVDF and $\mathrm{MgH}_{2}$ (Figure S5). By adopting PMMA as binder, the performance of GMH composite, however, is largely enabled. As shown in Figure 2a, the discharge capacity retains at $946 \mathrm{mAh}$ $\mathrm{g}^{-1}$ even after 100 cycles. Moreover, GMH-PMMA exhibits a good rate capability (Figure 2c). The average capacities are 1595, 1422, 1010, 726 and $589 \mathrm{mAh} \mathrm{g}^{-1}$ at 100, 200, 500, 1000 and $2000 \mathrm{~mA} \mathrm{~g}^{-1}$, respectively. When the current density returns to $100 \mathrm{~mA} \mathrm{~g}^{-1}$ after high-rate cycling, the capacity can restore to $1297 \mathrm{mAh} \mathrm{g}^{-1}$. Furthermore, a desirable long-term cycling performance at high current density of $2000 \mathrm{~mA} \mathrm{~g}^{-1}$ is measured (Figure 2d). After a slight fading in the initial 10 cycles, the reversible capacity retains $395 \mathrm{mAh} \mathrm{g}^{-1}$ at 1000 cycles, with a coulombic efficiency closes to $96.4 \%$, which is much better than the other samples measured under the same conditions (Figure S6). Although a slow decay of the capacity occurs at a high 
current density upon long cycling charge and discharge, the $\mathrm{MgH}_{2} / \mathrm{GR}$ composite enabled by PMMA binder still outperforms all the other $\mathrm{MgH}_{2}$-based electrodes reported in the literatures as shown in Table S1.

In addition, the loading ratios of $\mathrm{MgH}_{2}$ in the $\mathrm{GMH}$ composite were increased to $\sim 65 \mathrm{wt} \%$ and $80 \mathrm{wt} \%$ (denoted as GMH-65 and GMH-80, respectively) to investigate the effects of loading ratio on the lithium storage performance of the composite. Figure $\mathbf{S} 7$ presents the SEM images of the as-synthesized GMH-65 and GMH-80 and their cycling performances with PMMA binder at a current density of $100 \mathrm{~mA} \mathrm{~g}^{-1}$. It could be observed that, as the loading ratio of $\mathrm{MgH}_{2}$ rises, the specific capacity of GMH composite decreases compared with GMH-PMMA (50 wt\%), and the reversible specific capacity maintains at $705 \mathrm{mAh} \mathrm{g}^{-1}$ and $450 \mathrm{mAh} \mathrm{g}^{-1}$ for GMH-65 and GMH-80, respectively after 100 cycles. It is mainly ascribed to the possible agglomeration of $\mathrm{MgH}_{2}$ NPs with much higher density and less interparticle distance in the GMH-65 and GMH-80 compared with GMH, which will lower the overall conductivity of the composites, and hence reduce the cycling stability of the composite. Nonetheless, it should be clearly noticed that the cycling performance of GMH composite with a loading ratio as high as $80 \mathrm{wt} \%$ is still much better than the bulk composite. These results demonstrate a high accessibility for lithium insertion and extraction of $\mathrm{MgH}_{2} / \mathrm{GR}$, which are synergistically enhanced by the nanostructure of composites and the PMMA binder.

The lithiation/delithiation process of $\mathrm{MgH}_{2}$ has been widely researched, which can be described as the following equations: ${ }^{13,19}$

$$
\begin{aligned}
& \mathrm{MgH}_{2}+2 \mathrm{Li}^{+}+2 \mathrm{e}^{-} \leftrightarrow \mathrm{Mg}+2 \mathrm{LiH} \\
& \mathrm{Mg}+x \mathrm{Li}^{+}+x \mathrm{e}^{-} \leftrightarrow \mathrm{Li} x \mathrm{Mg}
\end{aligned}
$$


To better understand the conversion mechanism of GMH-PMMA, cyclic voltammetry (CV) measurements at a scan rate of $0.1 \mathrm{mV} \mathrm{s}^{-1}$ from $0.01 \mathrm{~V}$ to $3.0 \mathrm{~V}$ was performed (Figure 2e). In the first cycle, a large and broad cathodic peak centered at $0.59 \mathrm{~V}$ is mainly ascribed to the formation of SEI film, the conversion of $\mathrm{MgH}_{2}$, alone with the intercalation of $\mathrm{Li}$ into carbon; ${ }^{26,27}$ and the anodic peak at $\sim 0.78 \mathrm{~V}$ should correspond to the extraction of lithium and the regeneration of $\mathrm{MgH}_{2}$ of the composite (Equation 1) ${ }^{28}$. In the subsequent scans, the main cathodic peak shifts to $0.39 \mathrm{~V}$ while the anodic peak shows negligible modification, suggesting a stable SEI layer generated in the first cycle and a good reversibility of electrochemical reaction. The obtained CV curves is consistent with the voltage profiles of GMH composite (Figure 2b), which exhibits a slope discharge plateau during the first discharge process and a slant plateau in the charge process.

Electrochemical impedance spectroscopy (EIS) was used to confirm the enhanced kinetics of GMH composite. Each of the Nyquist plots of Figure $2 \mathrm{f}$ contains a depressed semicircle at medium and high frequencies, followed by a straight line with constant inclining angle at low frequencies. The equivalent circuit of GMH-PMMA is given in the inset of Figure 2f. Here, $R_{S}$, $R_{C T}, C P E$, and $W_{o}$ represent resistances related to SEI film, charge-transfer, constant phase elements, and Warburg impedance associated with lithium ion diffusion, respectively. It can be seen that GMH-PMMA displays a much smaller size of semicircle diameter and most inclined straight line, indicating a reduced decomposition of electrolyte, a low contact and charge-transfer resistance at electrode/electrolyte interface, a small diffusion resistance relating to lithium ions diffusion in electrode, and good capacitive behavior. ${ }^{29}$ Based on the fitting results, the $R_{C T}$ values of GMH-PMMA electrode are calculated to be $26.52 \Omega$ and $54.68 \Omega$ after 3 cycles and 100 cycles (Figure S8), respectively, while the corresponding value for GMH-BF increases 
largely from $28.99 \Omega$ to $134.5 \Omega$ due to the continuous formation of SEI film during cycling (Table S2). It demonstrates the stable interface reaction between the electrolyte and $\mathrm{MgH}_{2}$ during repeated lithiation and delithiation process due to the presence of PMMA binder, which leads to stable cycling performance. Those results further verify that the synergistic interactions between $\mathrm{MgH}_{2} \mathrm{NPs}$ and conductive GR of nanostructure ensure the superiority of composite; and meanwhile, the incorporation of PMMA plays a critical role in forming a stable SEI layer and facilitating rapid transfer of lithium ions, thus leading to a significantly enhanced reversible lithium storage, cycle stability and rate capability.

To gain a further mechanism comprehension of electrochemical performance of GMH composite, the HRTEM and XRD were conducted to monitor the active materials at full lithiation/delithiation states. Upon discharged to $0.001 \mathrm{~V}$ in the first cycle, the overall shape of electrode is well preserved with an obvious SEI films covered on surface (Figure 3a). Notably, resulting from the lithiation process (Equation 1), the obtained active nanoparticles are much transparent with slight size increase to around $16.2 \mathrm{~nm}$ due to the incorporation of lithium ions into $\mathrm{MgH}_{2}$ (Figure S9), and embedded in graphene layers uniformly (Figure 3b, c). The homogeneous distribution can also be depicted by the STEM image and the corresponding elemental mapping of $\mathrm{C}$ and $\mathrm{Mg}$ (Figure 3f). The HRTEM images and FFT patterns further confirm that there is almost no $\mathrm{MgH}_{2}$ detected and these generated nanocrystals are $\mathrm{LiH}$ and $\mathrm{Li}$ Mg alloys (Figure 3d, e). Specially, the marked lattice fringes with $d$-spacing of $2.04 \AA$ correspond to the (200) planes of $\mathrm{LiH}$, and the captured lattice fringes with $d$-spacing of $2.44 \AA$ and $2.58 \AA$ are in good agreement with that of the (101) and (002) planes of $\operatorname{Li}_{3} \mathrm{Mg}_{17}$, respectively. Those traits are consistent well with the corresponding SAED and FFT patterns (insets of Figure 3) and XRD results (Figure 3g). Moreover, when the electrode was charged 
back to $3.0 \mathrm{~V}$, diffraction peaks of $\mathrm{MgH}_{2}$ phases can be detected again, exhibiting good reversible conversion reactions. It is speculated that some other intermediate $\mathrm{Li}-\mathrm{Mg}$ phases (e.g., $\left.\mathrm{Li}_{3} \mathrm{Mg}_{7}\right)$ generated ${ }^{19}$ after being activated during cycles, and it is not clearly distinguished yet due to the poor crystallinity under the current condition.

Based on the above electrochemical performances, it is believed that the architecture of GMH as well as the strong particles-graphene interactions derived at nanoscale promises a high lithium storage capacity. For one thing, nano-structuring not only accelerate the electron/lithium diffusion rate due to the enlarged surface areas and shortened diffusion distances, but also relieve the strain induced by volume change; for another, the flexible and conductive GR nanosheets may serve as an extensive support to uniformly disperse $\mathrm{MgH}_{2} \mathrm{NPs}$, builds up highways for electron/lithium ion transport, and also plays a "flexible confinement" for buffering the strain induced by volume change during cycling. ${ }^{30-32}$

As mentioned above, the GMH-BF electrode demonstrates a high specific capacity in the first 20 cycles, but an apparent decline followed. However, this phenomenon can be largely altered by adopting the PMMA binder, which indicates that PMMA plays a critical role in further improving the cycling performance for GMH. It is believed that the mutual interfacial interactions between PMMA, $\mathrm{MgH}_{2} / \mathrm{Li}_{\mathrm{x}} \mathrm{Mg}$ nanocrystals and $\mathrm{GR}$, as well as the favorable lithium transfer/storage mechanism of PMMA during cycling are responsible for the enhanced performances.

To elaborate it, Figure 4a presents the schematic illustration of the interfacial interactions and lithium storage behaviors of the GMH-PMMA. Firstly, it is proposed that a larger number of ester functional groups of PMMA provide much more possible site to form the hydrogen bonding with $\mathrm{MgH}_{2} / \mathrm{Li}_{\mathrm{x}} \mathrm{Mg}$ NPs of GMH electrode. Specifically, particles at nanoscale and the 
large surface areas can yield a high volume fraction of the particle-polymer interfacial regions, and thus robust interfacial interactions can exist. ${ }^{33,} 34$ Besides, recent theoretical and experimental studies have proven that the interfacial interactions between PMMA and GR exhibit enhanced electrical conductivity and mechanical and thermal properties. ${ }^{33-35}$ More evidences can be found in the FTIR spectra (Figure $4 \mathrm{~b}$ ), which demonstrates a broad and strong peak at $\sim 3443 \mathrm{~cm}^{-1}$ assigned to a strong hydrogen bonding in comparison with PMMA spectrum; meanwhile, the typical absorptions in the stretching regions of PMMA at $\sim 1731 \mathrm{~cm}^{-1}$ $(\mathrm{C}=\mathrm{O})$ and $1272 \sim 1150 \mathrm{~cm}^{-1}$ (C-O-C) ${ }^{36,37}$ generate a red shift to $\sim 1635 \mathrm{~cm}^{-1}$ and $1232 \sim 1120$ $\mathrm{cm}^{-1}$, respectively. Those favorable interactions between PMMA and GMH composite not only ensure that active materials could be tightly attached to current collector, but also buffer the volume expansion and internal strain of active materials, which is beneficial to maintain the structural integrity of electrode. Thirdly, as to PMMA itself, it has been widely reported as binders, polymer electrolytes and separator for LIBs, because it helps to improve the rate of charge/lithium ion transfer and offer a beneficial conductivity environment. ${ }^{38-42}$ For one thing, PMMA exhibits high affinity toward liquid electrolytes at the presence of ester functional groups, which contributes to a high ionic conductivity and low interfacial impedance; for another, protons in the ester groups of PMMA molecule are highly active in coordinating with lithium ions. As a result, lithium ions can dissociate from their complexation sites and conjugate with new sites in electrolyte solution effectively. Specially, Yan Wang, et al. ${ }^{27}$ also propose a concept of lithium storage mechanism for a spin-coated PMMA thin film, and it demonstrates that PMMA can enhance both the reversible capacity and rate capability of electrodes as a rechargeable binder. 
The morphologies and nanostructure variations of GMH with and without PMMA during cycling were further characterized to confirm the structural stability of electrodes. Due to the present of PMMA, GMH after the first full cycle (Figure 4c) keeps the homogeneous morphology with active NPs uniformly anchored on GR. Such retention can be further confirmed by the corresponding SEM image (Figure S10a), which shows that active materials are wrapped by uniform SEI layer. Even after more cycles, the structure and morphology of GMH-PMMA is well preserved (Figure 4d, e), along with a uniform dispersion of $\mathrm{Mg}$ and $\mathrm{C}$ elements (Figure S11a), indicating a much stable and integrated structure of electrode. By comparison, the GMHBF electrode show a similar TEM (Figure 4f) and SEM (Figure S10d) images after the first cycle, however, agglomeration and cracks of active particles of electrode appear after 20 full cycles (Figure 4g). It is ascribed to the exposure of fresh $\mathrm{MgH}_{2}$ to the electrolyte due to the sever volume change during electrochemical reaction, leading to the continual reformation of the unstable SEI film coating on the $\mathrm{MgH}_{2}$ NPs, which results in rapid capacity decline. This phenomenon accumulates in the following cycles, and severe agglomeration or pulverization can be seen in electrode, with part of the active NPs even split away off the GR (Figure 4h and S10f). The quite different variations morphologies and structure during cycling further indicate the superiority and synergistic effect of PMMA binder in enabling the cycle stability of GMH composite.

\section{CONCLUSION}

In summary, we present a $\mathrm{MgH}_{2} / \mathrm{GR}$ composite with PMMA binder as anode material for LIBs. Monodisperse $\mathrm{MgH}_{2}$ NPs with an average particle size of $\sim 13.8 \mathrm{~nm}$ are bottom-up selfassembled uniformly on the ultrathin and flexible GR, forming a flexible interleaved architecture. Such architectures as well as the strong particles-graphene interactions derived at 
nanoscale result in significantly enhanced lithium storage capacity of $\mathrm{MgH}_{2} / \mathrm{GR}$ electrode. For one thing, nanosizing effect of $\mathrm{MgH}_{2} \mathrm{NPs}$ ensures highly favorable transport kinetics for both electrons and lithium ions of the whole electrode, as well as relieves the strain induced by volume change. For another, GR serves as an extensive support to uniformly disperse $\mathrm{MgH}_{2} \mathrm{NPs}$, builds up highways for electron/lithium ion transport, and plays a "flexible confinement" for buffering the strain caused by volume change during cycling. Moreover, the multifunctional PMMA, which demonstrates robust interfacial interactions with $\mathrm{MgH}_{2} / \mathrm{Li}_{\mathrm{x}} \mathrm{Mg}$ nanocrystals and GR of GMH composite, is proposed as a prospective binder for $\mathrm{MgH}_{2}$-based electrodes. It plays a critical role in maintaining the structural integrity of electrode, forming stable SEI films, and alleviating the volume expansion and detachment of active materials during cycling. As a result, benefiting from these synergistic effects of the nanostructure as well as the multifunctional PMMA binder, GMH composite is endowed with high reversible capacity, favorable rate capability and largely enhanced cyclability. The as-prepared electrodes deliver a high lithium storage capacity of $946 \mathrm{mAh} \mathrm{g}^{-1}$ at $100 \mathrm{~mA} \mathrm{~g}^{-1}$ over 100 full cycles, and good rate capability of $589 \mathrm{mAh} \mathrm{g}^{-1}$ even at a high current density of $2000 \mathrm{~mA} \mathrm{~g}^{-1}$. Therefore, the $\mathrm{MgH}_{2} / \mathrm{GR}$ composite enabled by PMMA binder proves to be a facile and promising candidate as anode material for high performance LIBs.

\section{EXPERIMENTAL SECTION}

Material Preparation. All chemicals were purchased from Sigma-Aldrich and used as received without further purification. In a typical experiment, $\mathrm{MgH}_{2} \mathrm{NPs}$ in-situ growth on GR with a loading ratio of $50 \mathrm{wt} . \%$ were fabricated via the hydrogenation of dibutyl magnesium $\left(\mathrm{MgBu}_{2}\right)$ in cyclohexane $\left(\mathrm{C}_{6} \mathrm{H}_{12}\right) .{ }^{43}$ Firstly, $\mathrm{MgBu}_{2}$ solution $(1.6 \mathrm{~mL}, 1 \mathrm{M}$ in heptane) and graphene $(31.2 \mathrm{mg})$ were mixed in cyclohexane $(40 \mathrm{~mL})$ in a pressure reactor vessel. The 
hydrogenation of $\mathrm{MgBu}_{2}$ was then carried out at $200{ }^{\circ} \mathrm{C}$ with vigorous stirring under a hydrogen pressure of 45 atm for $24 \mathrm{~h}$. Subsequently, the solution was centrifuged and dried at room temperature via dynamic vacuum on a Schlenk line, leading to the formation of GR-supported $\mathrm{MgH}_{2}$ NPs. Besides, $\mathrm{MgH}_{2} / \mathrm{GR}$ composite with higher loading ratios $(65 \mathrm{wt} \%$ and $80 \mathrm{wt} \%$, denoted as GMH-65 and GMH-80, respectively) were prepared for comparison.

Material Characterizations. The crystalline structures of samples were characterized by powder X-ray diffraction (XRD; D8 Advance, Bruker AXS) with $\mathrm{Cu} \mathrm{K} \alpha$ radiation. To prevent any possible reactions between samples and air during the XRD measurement, amorphous tape was used to cover the samples. FTIR spectra were recorded over 32 scans in absorption mode at a resolution of $4 \mathrm{~cm}^{-1}$ resolutions (Nicolet Nexus 470). Thermogravimetric analysis (TG; Netzsch STA 449 F3) was performed in air at a ramp rate of $5{ }^{\circ} \mathrm{C} \min ^{-1}$. The morphology and composition of samples were determined using an FE-SEM (JEOL 7500FA, Tokyo, Japan) and a TEM (JEOL 2011 F, Tokyo, Japan) coupled with an EDX spectrometer. Samples for TEM and SEM were firstly dispersed on $\mathrm{Cu}$ grids and conducting resin in glove box, respectively, and then rapidly transferred into the chambers for test in a few seconds. Specific surface area determinations were performed using the Brunauer-Emmett-Teller (BET) method with an adsorptionmeter (Quadrasorb SI-MP). Porosity was determined by BJH/DH method.

Electrode Fabrication. Electrochemical experiments were carried out with 2025 half-battery cells assembled with a pure lithium foil as counter electrode, Celgard 2340 as separator, and $1 \mathrm{M}$ $\mathrm{LiPF}_{6}$ in $\mathrm{EC} / \mathrm{DMC} / \mathrm{DEC}(1: 1: 1 \mathrm{v} / \mathrm{v} / \mathrm{v})$ solution as the electrolyte. The working electrodes were fabricated by coating a slurry consisting of active materials, acetylene black, and binders at a weight ratio of $8: 1: 1$, onto a nickel foam and dried in vacuum at $80{ }^{\circ} \mathrm{C}$ overnight. PMMA and PVDF binder was dissolved in N, N-dimethyl formamide (DMF). As comparison, electrodes 
without binder were fabricated under the same condition. The cells were assembled in an argonfilled glovebox with concentrations of moisture and oxygen below $0.1 \mathrm{ppm}$. The electrochemical capacity of composite was tested based on the weight of the active material $\left(\mathrm{MgH}_{2}\right)$ content. ${ }^{44}$

Electrochemical Measurements. Cyclic voltammetry (CV) at a scan rate of $0.1 \mathrm{mV} \mathrm{s}^{-1}$ in potential range of $0.01 \sim 3.0 \mathrm{~V}$ (vs. $\mathrm{Li}^{+} / \mathrm{Li}$ ) and electrochemical impedance spectroscopy (EIS) from $100 \mathrm{kHz}$ to $0.01 \mathrm{~Hz}$ were performed using a CHI660D electrochemistry workstation at room temperature. The cycling performance and rate stability of cells were tested using a Land Battery Test System between $0.001 \mathrm{~V}$ and $3.0 \mathrm{~V}$ (vs. $\mathrm{Li}^{+} / \mathrm{Li}$ ) at different constant current densities. In order to obtain reliable and reproductive results, at least three parallel cells were tested for each electrochemical measurement. Samples at different cycling states were collected from electrochemical cells and washed with DMC and THF in an argon-filled glovebox, and then dried overnight in vacuum for the XRD, TEM tests.

\section{ASSOCIATED CONTENT}

Supporting Information Available: Details about the additional SEM images, TEM images, the analysis of EDS, BET, TG, XRD results, and electrochemical performances of relevant samples. This material is available free of charge via the Internet at http://pubs.acs.org.

\section{AUTHOR INFORMATION}

\section{Corresponding Author}

*E-mail: yuxuebin@fudan.edu.cn; guanglin@uow.edu.au

\section{Author Contributions}

The manuscript was written through contributions of all authors. All authors have given approval to the final version of the manuscript. $\$$ These authors contributed equally. 


\section{ACKNOWLEDGMENT}

The authors would like to thank the financial support from the National Science Fund for

Distinguished Young Scholars (51625102), the National Natural Science Foundation of China (51471053) and the Science and Technology Commission of Shanghai Municipality (17XD1400700).

\section{REFERENCES}

(1) Wang, K.-X.; Li, X.-H.; Chen, J.-S. Surface and Interface Engineering of Electrode Materials for Lithium-Ion Batteries. Adv. Mater. 2015, 27, 527-545.

(2) Tang, Y.; Zhang, Y.; Li, W.; Ma, B.; Chen, X. Rational Material Design for Ultrafast Rechargeable Lithium-Ion Batteries. Chem. Soc. Rev. 2015, 44, 5926-5940.

(3) Croguennec, L.; Palacin, M. R. Recent Achievements on Inorganic Electrode Materials for Lithium-Ion Batteries. J. Am. Chem. Soc. 2015, 137, 3140-3156.

(4) Li, S.; Niu, J.; Zhao, Y. C.; So, K. P.; Wang, C.; Wang, C. A.; Li, J. High-Rate Aluminium Yolk-Shell Nanoparticle Anode for Li-Ion Battery with Long Cycle Life and Ultrahigh Capacity. Nat. Commun. 2015, 6, 7872.

(5) Chan, C. K.; Peng, H.; Liu, G.; McIIwrath, K.; Zhang, X. F.; Huggins, R. A.; Cui, Y. High-Performance Lithium Battery Anodes Using Silicon Nanowires. Nat. Nanotech. 2008, 3, $31-35$.

(6) Zhao, Y.; Li, X.; Yan, B.; Xiong, D.; Li, D.; Lawes, S.; Sun, X. Recent Developments and Understanding of Novel Mixed Transition-Metal Oxides as Anodes in Lithium Ion Batteries. Adv. Energy Mater. 2016, 6, 1502175 
(7) Youn, D. H.; Stauffer, S. K.; Xiao, P.; Park, H.; Nam, Y.; Dolocan, A. D.; Henkelman, G.; Heller, A.; Mullins, C. B. Simple Synthesis of Nanocrystalline Tin Sulfide/N-Doped Reduced Graphene Oxide Composites as Lithium Ion Battery Anodes. ACS Nano 2016, 10, 10778-10788.

(8) Xu, X.; Liu, W.; Kim, Y.; Cho, J. Nanostructured Transition Metal Sulfides for Lithium Ion Batteries: Progress and Challenges. Nano Today 2014, 9, 604-630.

(9) Zhu, J.; Sakaushi, K.; Clavel, G.; Shalom, M.; Antonietti, M.; Fellinger, T.-P. A General Salt-Templating Method to Fabricate Vertically Aligned Graphitic Carbon Nanosheets and Their Metal Carbide Hybrids for Superior Lithium Ion Batteries and Water Splitting. J. Am. Chem. Soc. 2015, 137, 5480-5485.

(10) Zhong, Y.; Xia, X.; Shi, F.; Zhan, J.; Tu, J.; Fan, H. J. Transition Metal Carbides and Nitrides in Energy Storage and Conversion. Adv. Sci. 2016, 3, 1500286.

(11) Li, G.-A.; Wang, C.-Y.; Chang, W.-C.; Tuan, H.-Y. Phosphorus-Rich Copper Phosphide Nanowires for Field-Effect Transistors and Lithium-Ion Batteries. ACS Nano 2016, 10, 86328644.

(12) Sun, M.; Liu, H.; Qu, J.; Li, J. Earth-Rich Transition Metal Phosphide for Energy Conversion and Storage. Adv. Energy Mater. 2016, 6, 1600087.

(13) Oumellal, Y.; Rougier, A.; Nazri, G. A.; Tarascon, J. M.; Aymard, L. Metal Hydrides for Lithium-Ion Batteries. Nat. Mater. 2008, 7, 916-921.

(14) Zeng, L.; Kawahito, K.; Ikeda, S.; Ichikawa, T.; Miyaoka, H.; Kojima, Y. Metal HydrideBased Materials towards High Performance Negative Electrodes for All-Solid-State Lithium-Ion Batteries. Chem. Commun. 2015, 51, 9773-9776. 
(15) Oumellal, Y.; Zlotea, C.; Bastide, S.; Cachet-Vivier, C.; Leonel, E.; Sengmany, S.; Leroy, E.; Aymard, L.; Bonnet, J.-P.; Latroche, M. Bottom-Up Preparation of $\mathrm{MgH}_{2}$ Nanoparticles with Enhanced Cycle Life Stability during Eelectrochemical Conversion in Li-Ion Batteries. Nanoscale 2014, 6, 14459-14466.

(16) Ikeda, S.; Ichikawa, T.; Kawahito, K.; Hirabayashi, K.; Miyaoka, H.; Kojima, Y. Anode Properties of Magnesium Hydride Catalyzed with Niobium Oxide for an All Solid-State Lithium-Ion Battery. Chem. Commun. 2013, 49, 7174-7176.

(17) Meggiolaro, D.; Gigli, G.; Paolone, A.; Reale, P.; Doublet, M. L.; Brutti, S. Origin of the Voltage Hysteresis of $\mathrm{MgH}_{2}$ Electrodes in Lithium Batteries. J. Phys. Chem. C 2015, 119, 17044-17052.

(18) Zhong, Y.; Yang, M.; Zhou, X.; Zhou, Z. Structural Design for Anodes of Lithium-Ion Batteries: Emerging Horizons from Materials to Electrodes. Mater. Horiz. 2015, 2, 553-566.

(19) Brutti, S.; Mulas, G.; Piciollo, E.; Panero, S.; Reale, P. Magnesium Hydride as a High Capacity Negative Electrode for Lithium Ion Batteries. J. Mater. Chem. 2012, 22, 14531-14537.

(20) Wang, Y.; Zheng, H.; Qu, Q.; Zhang, L.; Battaglia, V. S.; Zheng, H. Enhancing Electrochemical Properties of Graphite Anode by Using Poly(methylmethacrylate)-Poly (vinylidene fluoride) Composite Binder. Carbon 2015, 92, 318-326.

(21) Ling, M.; Qiu, J.; Li, S.; Yan, C.; Kiefel, M. J.; Liu, G.; Zhang, S. Multifunctional SAPProDOT Binder for Lithium Ion Batteries. Nano Lett. 2015, 15, 4440-4447. 
(22) Song, J.; Zhou, M.; Yi, R.; Xu, T.; Gordin, M. L.; Tang, D.; Yu, Z.; Regula, M.; Wang, D. Interpenetrated Gel Polymer Binder for High-Performance Silicon Anodes in Lithium-Ion Batteries. Adv. Funct. Mater. 2014, 24, 5904-5910.

(23) Kwon, T.-W.; Jeong, Y. K.; Lee, I.; Kim, T.-S.; Choi, J. W.; Coskun, A. Systematic Molecular-Level Design of Binders Incorporating Meldrum's Acid for Silicon Anodes in Lithium Rechargeable Batteries. Adv. Mater. 2014, 26, 7979-85.

(24) Zaidi, W.; Oumellal, Y.; Bonnet, J. P.; Zhang, J.; Cuevas, F.; Latroche, M.; Bobet, J. L.; Aymard, L. Carboxymethylcellulose and Carboxymethylcellulose-Formate as Binders in $\mathrm{MgH}_{2}-$ Carbon Composites Negative Electrode for Lithium-Ion Batteries. J. Power Sources 2011, 196, 2854-2857.

(25) Yang, Z.; Qian, K.; Lv, J.; Yan, W.; Liu, J.; Ai, J.; Zhang, Y.; Guo, T.; Zhou, X.; Xu, S et al. Encapsulation of $\mathrm{Fe}_{3} \mathrm{O}_{4}$ Nanoparticles into N, S Co-Doped Graphene Sheets with Greatly Enhanced Electrochemical Performance. Sci. Rep. 2016, 6, 27957.

(26) Huen, P.; Peru, F.; Charalambopoulou, G.; Steriotis, T. A.; Jensen, T. R.; Ravnsbæk, D. B. Nanoconfined $\mathrm{NaAlH}_{4}$ Conversion Electrodes for Li Batteries. ACS Omega 2017, 2, 1956-1967.

(27) Wang, Y.; Zhang, L.; Zhang, L.; Zhang, F.; He, P.; Zheng, H.; Zhou, H. Reversible Lithium-Ion Uptake in Poly(methylmethacrylate) Thin-Film via Lithiation/Delithiation at In Situ Formed Intramolecular Cyclopentanedione. Adv. Energy Mater. 2016, 6, 1601375.

(28) Peng, X.; Wang, H.; Hu, R.; Ouyang, L.; Liu, J.; Zhu, M. Electrochemical Performances of $\mathrm{MgH}_{2}$ and $\mathrm{MgH}_{2}-\mathrm{C}$ Films for Lithium Ion Battery Anode. J. Alloys Compd. 2017, 711, 473479. 
(29) Wang, Y.; Wang, C.; Wang, Y.; Liu, H.; Huang, Z. Superior Sodium-Ion Storage Performance of $\mathrm{Co}_{3} \mathrm{O}_{4} @$ Nitrogen-Doped Carbon: Derived from a Metal-organic Framework. $J$. Mater. Chem. A 2016, 4, 5428-5435.

(30) Yan, C.; Chen, G.; Zhou, X.; Sun, J.; Lv, C. Template-Based Engineering of CarbonDoped $\mathrm{Co}_{3} \mathrm{O}_{4}$ Hollow Nanofibers as Anode Materials for Lithium-Ion Batteries. Adv. Funct. Mater. 2016, 26, 1428-1436.

(31) Trogadas, P.; Ramani, V.; Strasser, P.; Fuller, T. F.; Coppens, M.-O. Hierarchically Structured Nanomaterials for Electrochemical Energy Conversion. Angew. Chem., Int. Ed. 2016, $55,122-148$.

(32) Luo, B.; Zhi, L. Design and Construction of Three Dimensional Graphene-Based Composites for Lithium Ion Battery Applications. Energy Environ. Sci. 2015, 8, 456-477.

(33) Fan, Z.; Gong, F.; Nguyen, S. T.; Duong, H. M. Advanced Multifunctional Graphene Aerogel-Poly (methyl methacrylate) Composites: Experiments and Modeling. Carbon 2015, 81, 396-404.

(34) Zhang, L.; Bai, Z.; Liu, L. Exceptional Thermal Conductance across Hydrogen-Bonded Graphene/Polymer Interfaces. Adv. Mater. Interfaces 2016, 3, 1600211.

(35) Pramoda, K. P.; Hussain, H.; Koh, H. M.; Tan, H. R.; He, C. B. Covalent Bonded Polymer-Graphene Nanocomposites. J. Polym. Sci., Part A: Polym. Chem. 2010, 48, 4262-4267.

(36) Jang, J.; Oh, J. H. Fabrication of a Highly Transparent Conductive Thin Film from Polypyrrole/Poly(methyl methacrylate) Core/Shell Nanospheres. Adv. Funct. Mater. 2005, 15, 494-502. 
(37) Kaczmarek, H.; Gałka, P.; Kowalonek, J. Influence of a Photoinitiator on the Photochemical Stability of Poly (methyl methacrylate) Studied with Fourier Transform Infrared Spectroscopy. J. Appl. Polym. Sci. 2010, 115, 1598-1607.

(38) Yu, X.; Jiang, A.; Yang, H.; Meng, H.; Dou, P.; Ma, D.; Xu, X. Facile Synthesis of Hollow Sn-Co@PMMA Nanospheres as High Performance Anodes for Lithium-Ion Batteries via Galvanic Replacement Reaction and In Situ Polymerization. Appl. Surf. Sci. 2015, 347, 624631.

(39) Sharma, R.; Sil, A.; Ray, S. Effect of Carbon Nanotube Dispersion on Electrochemical and Mechanical Characteristics of Poly (methyl methacrylate)-Based Gel Polymer Electrolytes. Polym. Compos. 2016, 37, 1936-1944.

(40) Meng, H.; Yang, H.; Yu, X.; Dou, P.; Ma, D.; Xu, X. Synthesis of Sn-Co@PMMA Nanowire Arrays by Electrodeposition and In Situ Polymerization as a High Performance Lithium-Ion Battery Anode. RSC Adv. 2015, 5, 95488-95494.

(41) Ali, U.; Karim, K. J. B. A; Buang, N. A. A Review of the Properties and Applications of Poly (Methyl Methacrylate) (PMMA). Polym. Rev. 2015, 55, 678-705.

(42) Liu, J.; Zhang, Q.; Zhang, T.; Li, J.-T.; Huang, L.; Sun, S.-G. A Robust Ion-Conductive Biopolymer as a Binder for Si Anodes of Lithium-Ion Batteries. Adv. Funct. Mater. 2015, 25, $3599-3605$.

(43) Xia, G. L.; Tan, Y.; Chen, X.; Sun, D.; Guo, Z.; Liu, H.; Ouyang, L.; Zhu, M.; Yu, X. Monodisperse Magnesium Hydride Nanoparticles Uniformly Self-Assembled on Graphene. Adv. Mater. 2015, 27, 5981-5988. 
(44) Li, W.; Yang, Z.; Li, M.; Jiang, Y.; Wei, X.; Zhong, X.; Gu, L.; Yu, Y. Amorphous Red

Phosphorus Embedded in Highly Ordered Mesoporous Carbon with Superior Lithium and Sodium Storage Capacity. Nano Lett. 2016, 16, 1546-1553. 


\section{FIGURES}
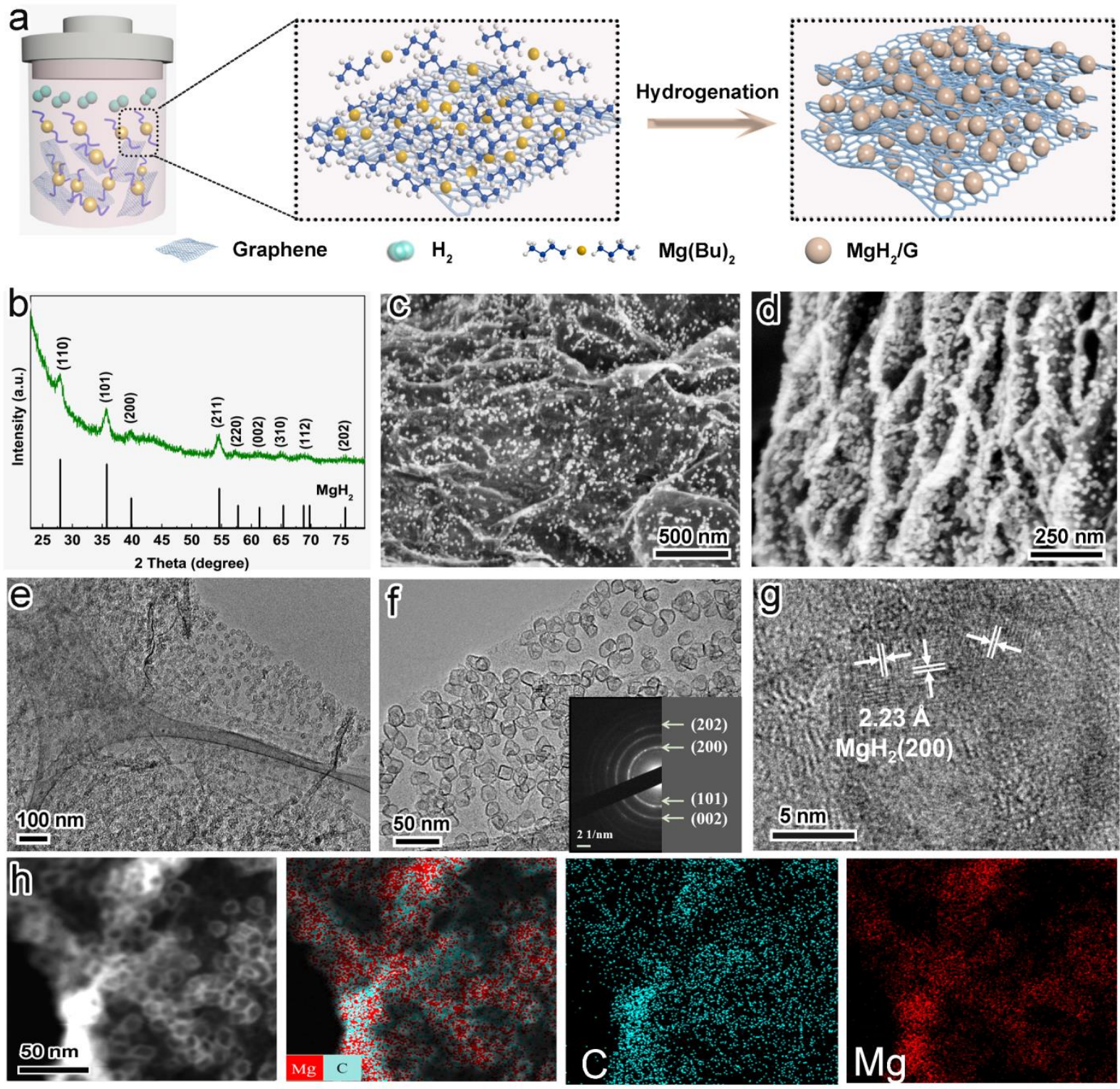

Figure 1. (a) Schematic illustration of the fabrication of GMH composite. (b) Typical XRD patterns of GMH. (c, d) SEM images of GMH. (e, f) TEM and (g) HRTEM images of GMH. (h) The STEM image. (i) The STEM image and the corresponding elemental mapping of $\mathrm{C}$ and $\mathrm{Mg}$. The inset of (f) is the corresponding SAED image. 

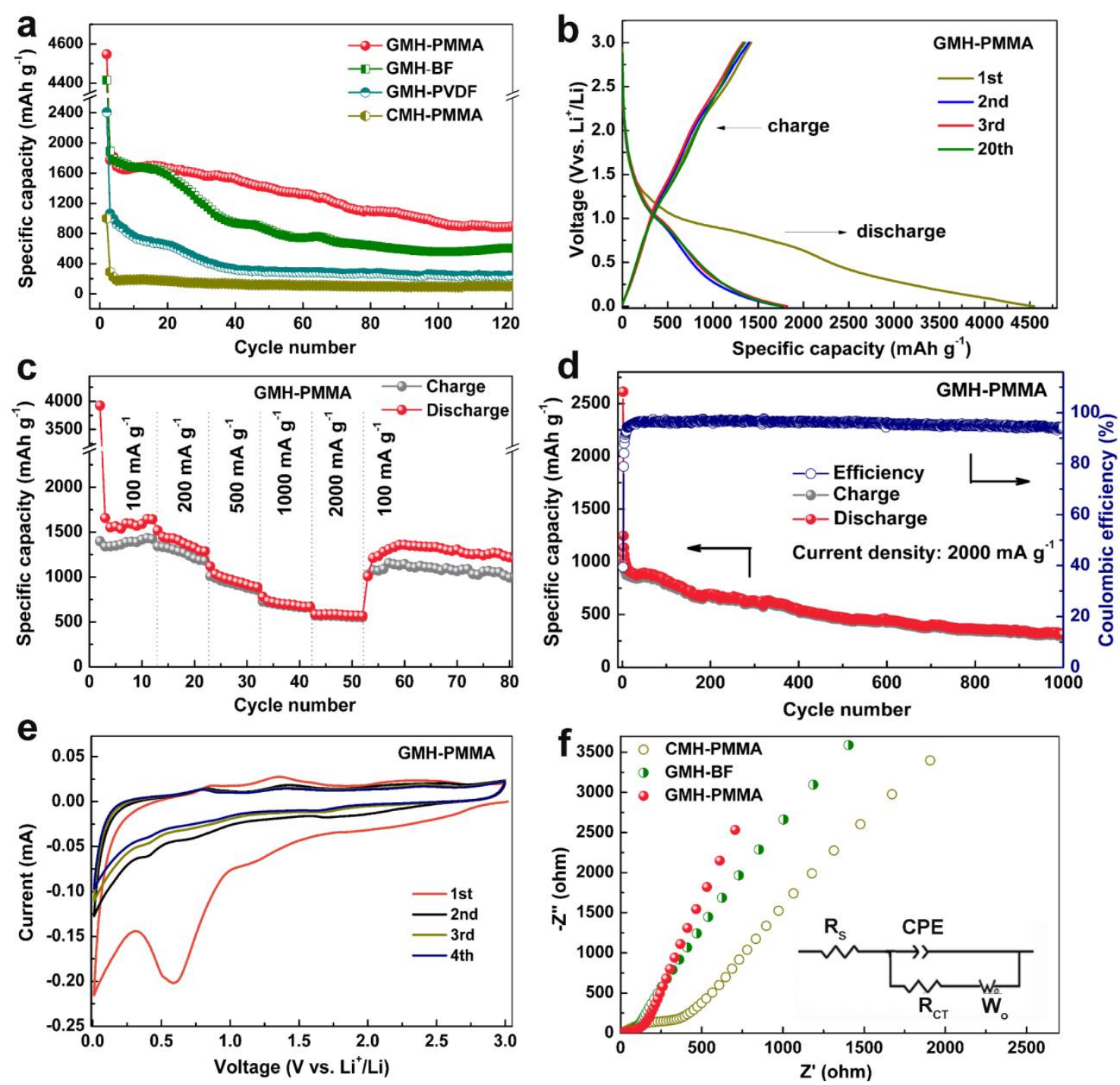

Figure 2. (a) Cycling performances of the GMH-PMMA, GMH-BF, GMH-PVDF, and CMHPMMA electrodes cycled at $100 \mathrm{~mA} \mathrm{~g}^{-1}$. (b) Discharge and charge voltage profiles of GMHPMMA electrode cycled at a current of $100 \mathrm{~mA} \mathrm{~g}^{-1}$. (c) Rate capacity of GMH-PMMA at various current rates from 100 to $2000 \mathrm{~mA} \mathrm{~g}^{-1}$. (d) Cycling performance of GMH-PMMA at a high current density of $2000 \mathrm{~mA} \mathrm{~g}^{-1}$. (e) $\mathrm{CV}$ profiles of GMH-PMMA at a scan rate of $0.1 \mathrm{mV}$ $\mathrm{s}^{-1}$. (f) EIS of the CMH-PMMA, GMH-BF, and GMH-PMMA from $100 \mathrm{kHz}$ to $0.01 \mathrm{~Hz}$ after $\mathrm{CV}$ test (insets: the equivalent circuit). All carried out in the voltage range $3.0-0.001 \mathrm{~V}$ (vs. Li ${ }^{+} / \mathrm{Li}$ ). The capacities here are tested based on the weight percentage of $\mathrm{MgH}_{2}$. 

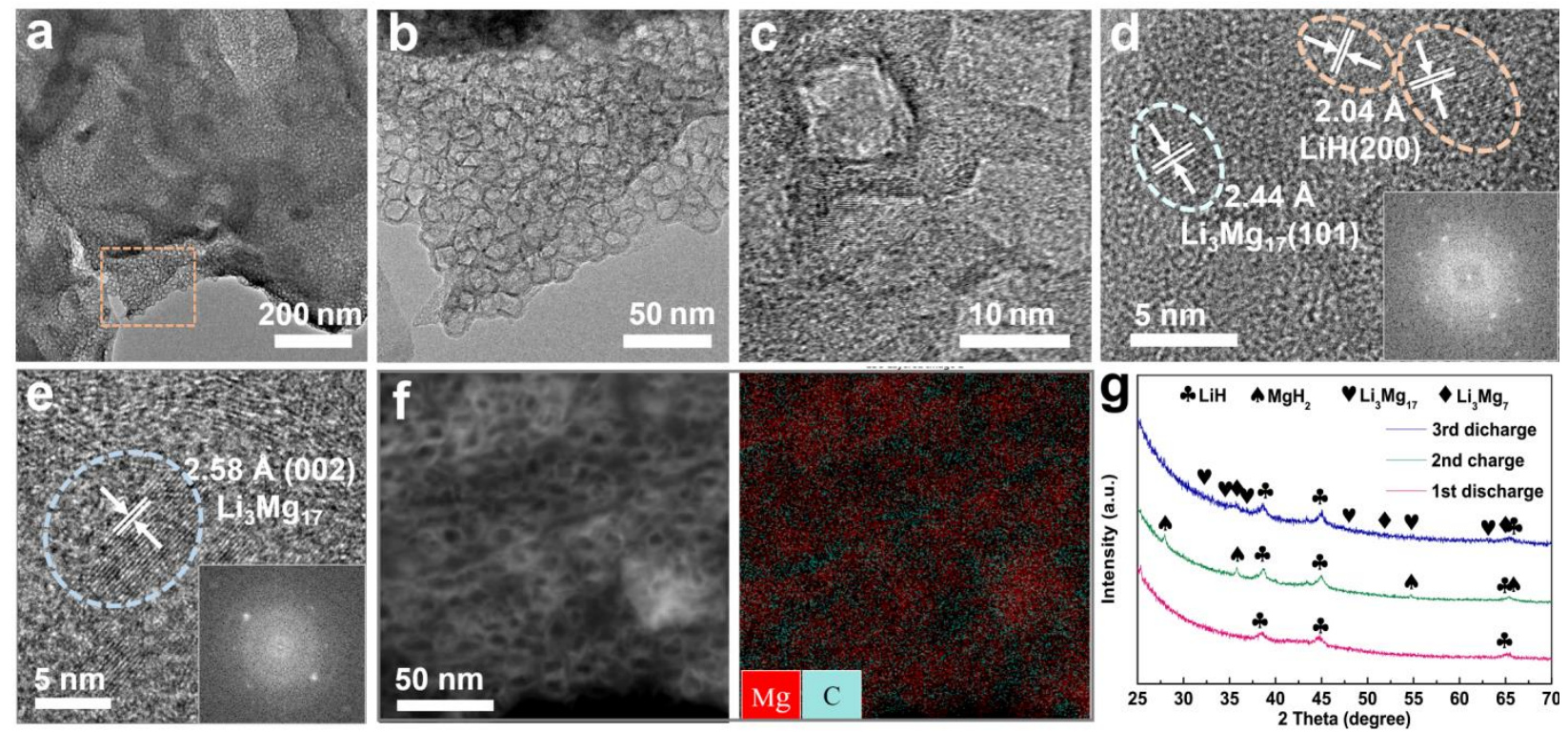

Figure 3. (a, b) TEM images and (c, d, e) HRTEM images, (f) STEM image and the corresponding elemental mapping of $\mathrm{C}, \mathrm{Mg}$ of GMH-PMMA at first full discharged state. The insets of $(\mathrm{a}, \mathrm{d}, \mathrm{e})$ are the corresponding SAED and FFT patterns. (g) XRD patterns of GMHPMMA at different cycle states (the red line: the first full discharged; the green line: the first full charged; the blue line: the second full discharged). 

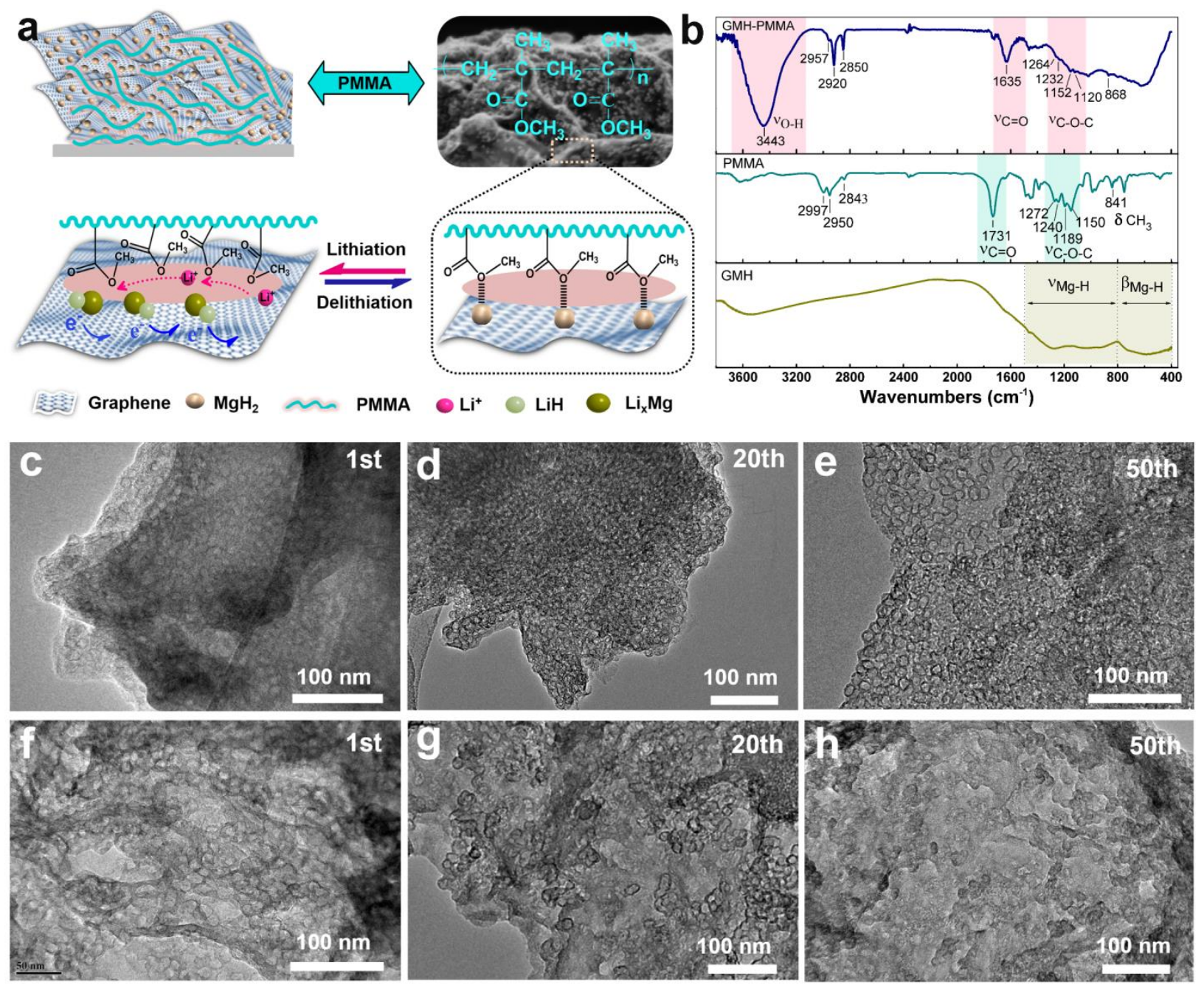

Figure 4. (a) Schematic illustration of the interfacial interactions and lithium storage behaviour of GMH-PMMA. (b) FTIR spectra of the GMH-PMMA, pure PMMA, and GMH samples. (c, d,

e) TEM images of the GMH-PMMA at discharged state after 1st, 20th, 50th cycles at $100 \mathrm{~mA} \mathrm{~g}^{-}$

${ }^{1}$. (f, g, h) TEM images of GMH-BF at discharged state after 1st, 20th, 50th cycles at $100 \mathrm{~mA} \mathrm{~g}^{-}$ 1. 
Table of Contents

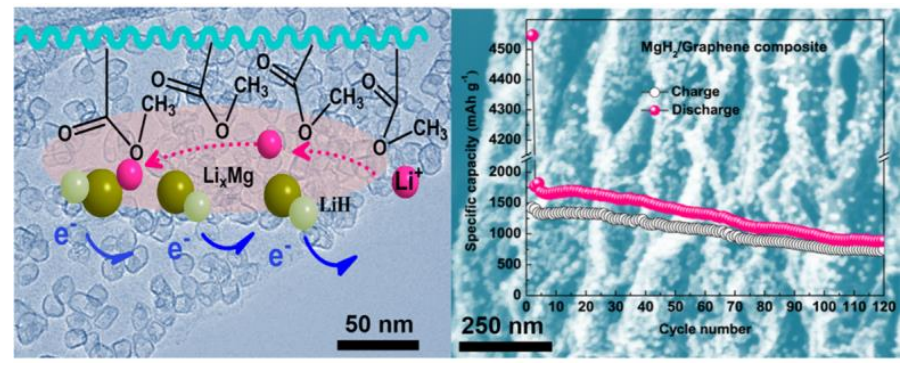

